\title{
Analytical Predictions of Period-1 motions to Chaos in a Periodically Driven Quadratic Nonlinear Oscillator with a Time-delay
}

\author{
A.C.J $\mathrm{Luo}^{1 *}, \mathrm{~S} . \mathrm{Xing}^{2}$ \\ Department of Mechanical and Industry Engineering \\ Southern Illinois University Edwardsville, \\ Edwardsville, IL-62026, USA
}

\begin{abstract}
In this paper, periodic motions in a periodically forced, damped, quadratic nonlinear oscillator with time-delayed displacement are analytically predicted through implicit discrete mappings of the corresponding differential equation. From mapping structures, bifurcation trees of periodic motions are achieved analytically, and the corresponding stability and bifurcation analysis are carried out through eigenvalue analysis. From the analytical prediction, numerical results of periodic motions are illustrated to verify such an analytical prediction. The semianalytical method gives the analytical prediction of the periodic motions matching very well with the approximate analytical solution for the time-delayed, quadratic nonlinear system. The method can also be applied to other time-delayed nonlinear systems.
\end{abstract}

Keywords and phrases: Time-delay quadratic nonlinear oscillator, Period-1 motions to chaos, Bifurcation tree, Implicit mapping, Mapping structures

Mathematics Subject Classification: 34A34, 34B20, 34C15

\section{Introduction}

Periodic solutions in nonlinear dynamical systems have been of great interest for a long time. Until now, one cannot find a suitable method to give reasonable results. In 1788, Lagrange [1] investigated periodic motions of three-body problem through a perturbation of the two-body problem with the method of averaging. In the end of 19th century, Poincare [2] developed the perturbation theory for periodic motions of celestial bodies. In 1920, van der Pol [3] employed the method of averaging for the periodic solutions of oscillation systems in circuits. Until 1928, the asymptotic validity of the method of averaging was not proved. Fatou [4] gave the proof of the asymptotic validity through the solution existence theorems of differential equations. In 1935, Krylov and Bogoliubov [5] further developed the method of averaging for nonlinear oscillations in nonlinear vibration systems. Since then, researchers extensively used the perturbation method to investigate periodic solutions in nonlinear dynamical systems. In 2012, Luo [6] developed an analytical method for analytical solutions of periodic motions in nonlinear dynamical

*Corresponding author. E-mail: aluo@siue.edu 
systems. Luo and Huang [7] applied this method to the Duffing oscillator for finding approximate solutions of periodic motions, and Luo and Huang [8] gave the analytical bifurcation trees of period-m motions to chaos in the Duffing oscillator (also see, Luo and Huang $[9,10]$ ).

In 2013, Luo [11] systematically proposed an analytical method to obtain the analytical solution of periodic motions in time-delayed, nonlinear dynamical systems. Luo and Jin [12] used such an analytical method to determine the analytical bifurcation trees of period- 1 motions to chaos in time-delayed, quadratic nonlinear oscillator. Luo and Jin [13] investigated complex period-1 motions of the periodically forced Duffing oscillator with a time-delayed displacement, which cannot be obtained from the traditional harmonic balance and perturbation methods. Luo and Jin [14] analytically investigated the period-m motions of the time-delayed Duffing oscillator. The corresponding complex period-m motions were obtained for the first time, and the bifurcation trees of period-1 motion to chaos were also discussed.

Through discretization, discrete maps for dynamical systems can be developed. Based on nonlinear maps, periodic motions of continuous dynamical systems can be investigated. In 2005, Luo [15] presented a mapping dynamics of discrete dynamical systems to determime periodic motions. The systematical discussion of mapping dynamics in discontinuous dynamical systems was presented in Luo [16]. The discrete maps can be implicit or explicit functions. From discrete mapping structures, periodic motions in discrete dynamical systems can be predicted analytically, and the stability and bifurcation analysis of periodic motions in nonlinear dynamical systems can be carried out. To determine periodic motions in continuous dynamical systems, in 2014, Luo [17] developed a semi-analytical method to determine periodic motions in nonlinear dynamical systems through discrete implicit maps. Luo and Guo [18] applied this approach to investigate bifurcation tree of a Duffing oscillator. In this paper, the semianalytical method will be used to investigate the bifurcation of a periodically forced, quadratic nonlinear oscillator with time-delay, and the corresponding methodology will be presrented herein.

In this paper, periodic motions in the quadratic nonlinear oscillator with time-delay will be investigated through the mapping structures of discrete implicit maps. From mapping structures, periodic motions will be predicted analytically, and the corresponding stability and bifurcation of periodic motions will be analyzed. The bifurcation tree of periodic motion to chaos will be investigated. Numerical results of periodic motions will be performed. Furthermore, complex nonlinear behaviors in such a time-delayed dynamical systems will be discussed.

\section{Discrete mappings}

In this paper, consider a quadratic nonlinear oscillator with time delay as

$$
\ddot{x}+\delta \dot{x}+\alpha_{1} x-\alpha_{2} x^{\tau}+\beta x^{2}=Q_{0} \cos \Omega t
$$

where $x=x(t)$ and $x^{\tau}=x(t-\tau)$. The above equation becomes

$$
\begin{aligned}
& \dot{x}=y, \\
& \dot{y}=Q_{0} \cos \Omega t-\delta y-\alpha_{1} x+\alpha_{2} x^{\tau}-\beta x^{2}
\end{aligned}
$$

Setting $\mathbf{x}=(x, y)^{T}$, from Luo[15], the differential equation in Eq.(2.2) can be discretized by a midpoint scheme for the time interval $t \in\left[t_{k-1}, t_{k}\right]$ to form an implicit map $P_{k}(k=1,2, \ldots)$ as

$$
\begin{aligned}
& P_{k}:\left(\mathbf{x}_{k-1}, \mathbf{x}_{k-1}^{\tau}\right) \rightarrow\left(\mathbf{x}_{k}, \mathbf{x}_{k}^{\tau}\right) \\
\Rightarrow & \left(\mathbf{x}_{k}, \mathbf{x}_{k}^{\tau}\right)=P_{k}\left(\mathbf{x}_{k-1}, \mathbf{x}_{k-1}^{\tau}\right)
\end{aligned}
$$

with the implicit relations

$$
\begin{aligned}
x_{k} & =x_{k-1}+\frac{1}{2} h\left(y_{k}+y_{k-1}\right) \\
y_{k} & =y_{k-1}+h\left[Q_{0} \cos \Omega\left(t_{k-1}+\frac{h}{2}\right)-\frac{1}{2} \delta\left(y_{k}+y_{k-1}\right)\right. \\
& \left.-\frac{1}{2} \alpha_{1}\left(x_{k}+x_{k-1}\right)+\frac{1}{2} \alpha_{2}\left(x_{k}^{\tau}+x_{k-1}^{\tau}\right)-\frac{1}{4} \beta\left(x_{k}+x_{k-1}\right)^{2}\right]
\end{aligned}
$$


The time delay nodes $\mathbf{x}_{k}^{\tau} \approx \mathbf{x}\left(t_{k}-\tau\right)$ of $\mathbf{x}_{k} \approx \mathbf{x}(t)$ will lie between $\mathbf{x}_{k-l_{k}}$ and $\mathbf{x}_{k-l_{k}-1}\left(l_{k}=\operatorname{int}(\tau / h)\right)$, which can be expressed by an interpolation function with two points $\mathbf{x}_{k-l_{k}}$ and $\mathbf{x}_{k-l_{k}-1}$. Thus the two time-delay nodes can be expressed by

$$
\left.\begin{array}{l}
\mathbf{x}_{j}^{\tau}=\mathbf{h}_{j}\left(\mathbf{x}_{r_{j}-1}, \mathbf{x}_{r_{j}}, \theta_{r_{j}}\right) \\
\theta_{r_{j}}=\frac{\left(t_{j}-\tau\right)-t_{r_{j-1}}}{t_{r_{j}}-t_{r_{j-1}}} \text { and } r_{j}=j-l_{j} \\
(j=k, k-1)
\end{array}\right\}
$$

By using the simple Lagrange interpolation, the time-delay nodes $\mathbf{x}_{j}^{\tau}=\mathbf{h}_{j}\left(\mathbf{x}_{r_{j}-1}, \mathbf{x}_{r_{j}}, \theta_{r j}\right)$ can be written as

$$
\begin{aligned}
& x_{j}^{\tau}=\left(x_{j-l_{j}-1}\right)+\left(1-\frac{\tau}{h}+l_{j}\right)\left(x_{j-l_{j}}-x_{j-l_{j}-1}\right) \\
& y_{j}^{\tau}=\left(y_{j-l_{j}-1}\right)+\left(1-\frac{\tau}{h}+l_{j}\right)\left(y_{j-l_{j}}-y_{j-l_{j}-1}\right)
\end{aligned}
$$

Thus, the time-delay nodes are expressed by non-time-delay nodes.

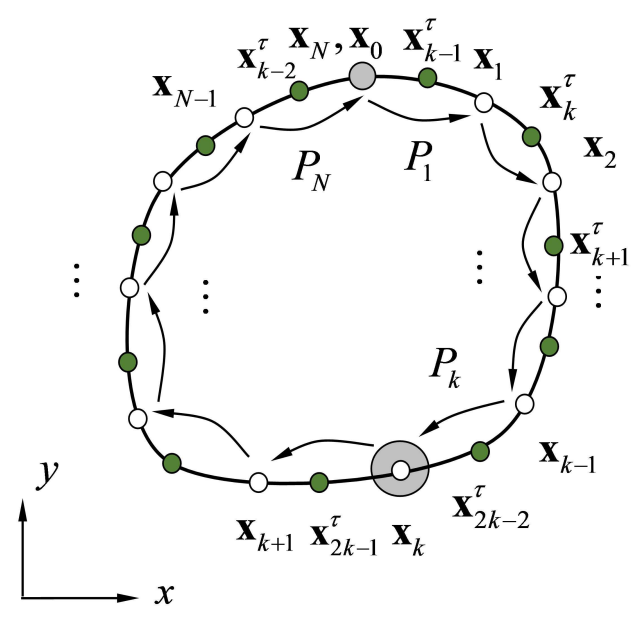

FIgURE 1. Mapping structure of period-1 motion with N non-time-delayed nodes and $\mathrm{N}$ time-delayed nodes.

\section{Periodic motions}

In general, a period-m motion in such a quadratic nonlinear oscillator can be represented by a discrete mapping structure as follows:

$$
\begin{gathered}
P=\underbrace{P_{m N} \circ P_{m N-1} \circ \cdots \circ P_{2} \circ P_{1}}_{m N-\text { actions }}:\left(\mathbf{x}_{0}^{(m)}, \mathbf{x}_{0}^{\tau(m)}\right) \rightarrow\left(\mathbf{x}_{m N}^{(m)}, \mathbf{x}_{m N}^{\tau(m)}\right) \\
\Rightarrow\left(\mathbf{x}_{m N}^{(m)}, \mathbf{x}_{m N}^{\tau(m)}\right)=P\left(\mathbf{x}_{0}^{(m)}, \mathbf{x}_{0}^{\tau(m)}\right)
\end{gathered}
$$

with

$$
\begin{aligned}
& P_{k}:\left(\mathbf{x}_{k-1}^{(m)}, \mathbf{x}_{k-1}^{\tau(m)}\right) \rightarrow\left(\mathbf{x}_{k}^{(m)}, \mathbf{x}_{k}^{\tau(m)}\right) \\
& (k=1,2, \cdots, m N) .
\end{aligned}
$$




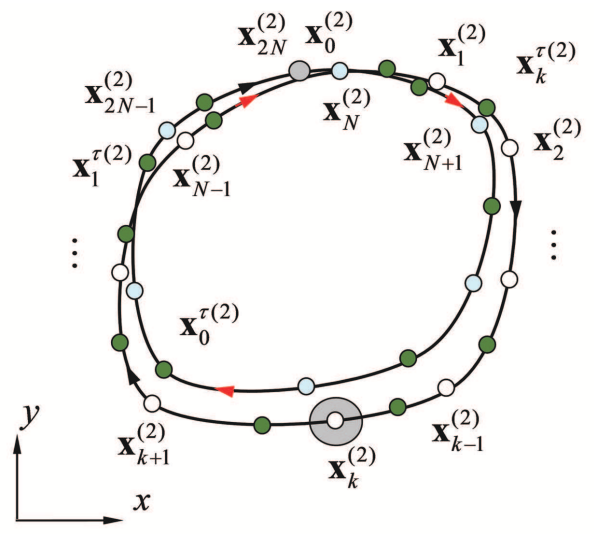

FIgURE 2. Mapping structure of period-2 motion with 2N non-time-delayed nodes and $2 \mathrm{~N}$ time-delayed nodes.

The set of points $\mathbf{x}_{k}^{(m) *}$ on the periodic motion for the time-delayed nonlinear system is computed by

$$
\left.\begin{array}{l}
\mathbf{g}_{k}\left(\mathbf{x}_{k-1}^{(m) *}, \mathbf{x}_{k}^{(m) *}, \mathbf{x}_{k-1}^{\tau(m) *}, \mathbf{x}_{k}^{\tau(m) *}, \mathbf{p}\right)=\mathbf{0} \\
\mathbf{x}_{j}^{\tau(m) *}=\mathbf{h}_{j}\left(\mathbf{x}_{r_{j}-1}^{(m)}, \mathbf{x}_{r_{j}}^{(m) *}, \theta_{r_{j}}\right),(j=k, k-1)
\end{array}\right\}(k=1,2, \cdots, m N)
$$

From Eq.(2.4), the corresponding algebraic equations can be obtained. i.e.

$$
\begin{aligned}
x_{k}^{(m)} & =x_{k-1}^{(m)}+\frac{1}{2} h\left(y_{k}^{(m)}+y_{k-1}^{(m)}\right), \\
y_{k}^{(m)} & =y_{k-1}^{(m)}+h\left[Q_{0} \cos \Omega\left(t_{k-1}+\frac{1}{2} h\right)-\frac{1}{2} \delta\left(y_{k}^{(m)}+y_{k-1}^{(m)}\right)\right. \\
& \left.-\frac{1}{2} \alpha_{1}\left(x_{k}^{(m)}+x_{k-1}^{(m)}\right)+\frac{1}{2} \alpha_{2}\left(x_{k}^{\tau(m)}+x_{k-1}^{\tau(m)}\right)-\frac{1}{4} \beta\left(x_{k}^{(m)}+x_{k-1}^{(m)}\right)^{2}\right] \\
& (k=1,2, \cdots, m N)
\end{aligned}
$$

From Eq.(2.6), the corresponding algebraic equations are

$$
\left.\begin{array}{l}
x_{j}^{\tau(m)}=x_{j-l_{j}-1}^{(m)}+\left(1-\frac{\tau}{h}+l_{j}\right)\left(x_{j-l_{j}}^{(m)}-x_{j-l_{j}-1}^{(m)}\right) \\
y_{j}^{\tau(m)}=y_{j-l_{j}-1}^{(m)}+\left(1-\frac{\tau}{h}+l_{j}\right)\left(y_{j-l_{j}}^{(m)}-y_{j-l_{j}-1}^{(m)}\right)
\end{array}\right\}(j=k, k-1) .
$$

The discrete node points $\mathbf{x}_{k}=\left(x_{k}, y_{k}\right)^{T}$ and $\mathbf{x}_{k}^{\tau}=\left(x_{k}^{\tau}, y_{k}^{\tau}\right)^{T}(k=0,1,2, \ldots)$ are used to illustrate period-1 and period-2 motions in Figs.1 and 2, respectively. The green points are for time-delay nodes. The white and blue points are for non-time-delay nodes. From Eqs.(2.6) and (3.1), node values of the discretized quadratic nonlinear oscillator can be determined by $2(m N+1)$ equations. Once the node points $x_{k}^{(m) *}(k=1,2, \ldots, m N)$ of the period-m motion are obtained, the stability of the period-m motion can be discussed by the corresponding Jacobian matrix. In vicinity of $\mathbf{x}_{k}^{(m) *}$ and $\mathbf{x}_{k}^{\tau(m) *}$, with $\mathbf{x}_{k}^{(m)}=\mathbf{x}_{k}^{(m) *}+\Delta \mathbf{x}_{k}^{(m)}$ and $\mathbf{x}_{k}^{\tau(m)}=\mathbf{x}_{k}^{\tau(m) *}+\Delta \mathbf{x}_{k}^{\tau(m)}$, the linearized equation is given by

$$
\begin{aligned}
& \sum_{j=k-1}^{k} \frac{\partial \mathbf{g}_{k}}{\partial \mathbf{x}_{j}^{(m)}} \Delta \mathbf{x}_{j}^{(m)}+\frac{\partial \mathbf{g}_{k}}{\partial \mathbf{x}_{j}^{\tau(m)}}\left(\frac{\partial \mathbf{x}_{j}^{\tau(m)}}{\partial \mathbf{x}_{r_{j}}^{(m)}} \Delta \mathbf{x}_{r_{j}}^{(m)}+\frac{\partial \mathbf{x}_{j}^{\tau(m)}}{\partial \mathbf{x}_{r_{j}-1}^{(m)}} \Delta \mathbf{x}_{r_{j}-1}^{(m)}\right)=\mathbf{0} \\
& \text { with } r_{j}=j-l_{j}, j=k-1, k(k=1,2, \cdots, m N) .
\end{aligned}
$$


Define

$$
\begin{aligned}
& \mathbf{y}_{k}^{(m)}=\left(\mathbf{x}_{k}^{(m)}, \mathbf{x}_{k-1}^{(m)}, \cdots, \mathbf{x}_{r_{k-1}}^{(m)}\right)^{\mathrm{T}}, \\
& \mathbf{y}_{k-1}^{(m)}=\left(\mathbf{x}_{k-1}^{(m)}, \mathbf{x}_{k-2}^{(m)}, \cdots, \mathbf{x}_{r_{k-1}-1}^{(m)}\right)^{\mathrm{T}}, \\
& \Delta \mathbf{y}_{k}^{(m)}=\left(\Delta \mathbf{x}_{k}^{(m)}, \Delta \mathbf{x}_{k-1}^{(m)}, \cdots, \Delta \mathbf{x}_{r_{k-1}}^{(m)}\right)^{\mathrm{T}}, \\
& \Delta \mathbf{y}_{k-1}^{(m)}=\left(\Delta \mathbf{x}_{k-1}^{(m)}, \Delta \mathbf{x}_{k-2}^{(m)}, \cdots, \Delta \mathbf{x}_{r_{k-1}-1}^{(m)}\right)^{\mathrm{T}} .
\end{aligned}
$$

The resultant Jacobian matrix of the periodic flow is

$$
D P=D P_{m N(m N-1) \ldots 1}=\left[\frac{\partial \mathbf{y}_{m N}^{(m)}}{\partial \mathbf{y}_{0}^{(m)}}\right]_{\left(\mathbf{y}_{0}^{(m) *}, \ldots, \mathbf{y}_{m N}^{(m)}\right)}
$$

where

$$
\Delta \mathbf{y}_{k}^{(m)}=\mathbf{A}_{k}^{(m)} \Delta \mathbf{y}_{k-1}^{(m)}, \mathbf{A}_{k}^{(m)} \equiv\left[\frac{\partial \mathbf{y}_{k}^{(m)}}{\partial \mathbf{y}_{k-1}^{(m)}}\right]_{\left(\mathbf{y}_{k-1}^{(m) *}, \mathbf{y}_{k}^{(m) *}\right)}
$$

and

$$
\begin{aligned}
& \mathbf{a}_{k j}^{(m)}=\left[\frac{\partial \mathbf{g}_{k}}{\partial \mathbf{x}_{k}^{(m)}}\right]^{-1} \frac{\partial \mathbf{g}_{k}}{\partial \mathbf{x}_{j}^{(m)}}, \\
& \mathbf{a}_{k r_{j}}^{(m)}=\left[\frac{\partial \mathbf{g}_{k}}{\partial \mathbf{x}_{k}^{(m)}}\right]^{-1} \frac{\partial \mathbf{g}_{k}}{\partial \mathbf{x}_{j}^{\tau(m)}} \frac{\partial \mathbf{x}_{j}^{\tau(m)}}{\partial \mathbf{x}_{r_{j}}^{(m)}}, \\
& \mathbf{a}_{k\left(r_{j}-1\right)}^{(m)}=\left[\frac{\partial \mathbf{g}_{k}}{\partial \mathbf{x}_{k}^{(m)}}\right]^{-1} \frac{\partial \mathbf{g}_{k}}{\partial \mathbf{x}_{j}^{\tau(m)}} \frac{\partial \mathbf{x}_{j}^{\tau(m)}}{\partial \mathbf{x}_{r_{j}-1}^{(m)}} \\
& \text { with } r_{j}=j-l_{j}, j=k-1, k ;
\end{aligned}
$$

$$
\begin{aligned}
& \mathbf{A}_{k}^{(m)}=\left[\begin{array}{cc}
\mathbf{B}_{k}^{(m)}\left(\mathbf{a}_{k\left(r_{k-1}-1\right)}^{(m)}\right)_{2 \times 2} \\
\mathbf{I}_{k}^{(m)} & \mathbf{0}_{k}^{(m)}
\end{array}\right]_{2(s+1) \times 2(s+1)}, s=1+l_{k-1} \\
& \mathbf{B}_{k}^{(m)}=\left[\left(\mathbf{a}_{k(k-1)}^{(m)}\right)_{2 \times 2}, \mathbf{0}_{2 \times 2}, \cdots,\left(\mathbf{a}_{k r_{k}}^{(m)}\right)_{2 \times 2},\left(\mathbf{a}_{k r_{k-1}}^{(m)}+\mathbf{a}_{k\left(r_{k}-1\right)}^{(m)}\right)_{2 \times 2}\right], \\
& \mathbf{I}_{k}^{(m)}=\operatorname{diag}\left(\mathbf{I}_{2 \times 2}, \mathbf{I}_{2 \times 2}, \cdots, \mathbf{I}_{2 \times 2}, \mathbf{I}_{2 \times 2}\right)_{2 s \times 2 s}, \\
& \mathbf{0}_{k}^{(m)}=(\underbrace{\mathbf{0}_{2 \times 2}, \mathbf{0}_{2 \times 2}, \cdots, \mathbf{0}_{2 \times 2}, \mathbf{0}_{2 \times 2}}_{s})^{\mathrm{T}} ;
\end{aligned}
$$

with

$$
\begin{aligned}
& \frac{\partial \mathbf{g}_{k}}{\partial \mathbf{x}_{k-1}^{(m)}}=\left[\begin{array}{cc}
-1 & -\frac{1}{2} h \\
\Delta & \frac{1}{2} \delta h-1
\end{array}\right], \frac{\partial \mathbf{g}_{k}}{\partial \mathbf{x}_{k}^{(m)}}=\left[\begin{array}{cc}
1 & -\frac{1}{2} h \\
\Delta & \frac{1}{2} \delta h+1
\end{array}\right], \\
& \frac{\partial \mathbf{x}_{j}^{\tau(m)}}{\partial \mathbf{x}_{r_{j}-1}^{(m)}}=\left[\begin{array}{cc}
0 & 0 \\
\frac{\tau}{h}-l_{j} & 0
\end{array}\right], \frac{\partial \mathbf{x}_{j}^{\tau(m)}}{\partial \mathbf{x}_{r_{j}}^{(m)}}=\left[\begin{array}{cr}
0 & 0 \\
1-\frac{\tau}{h}+l_{j} & 0
\end{array}\right], \\
& \frac{\partial \mathbf{g}_{k}}{\partial \mathbf{x}_{j}^{\tau(m)}}=\left[\begin{array}{cc}
0 & 0 \\
0 & -\frac{1}{2} h \alpha_{2}
\end{array}\right], \\
& \Delta=\frac{1}{2} h\left[\alpha_{1}+\beta\left(x_{k}+x_{k-1}\right)\right] .
\end{aligned}
$$

The eigenvalues for such a periodic flow are determined by

$$
\left|D P-\lambda \mathbf{I}_{2(s+1) \times 2(s+1)}\right|=0,
$$


(i) If the magnitudes of all eigenvalues of $D P$ are less than one (i.e., $\left|\lambda_{i}\right|<1, i, j \in 1,2, \ldots, 2(s+1)$ ), the approximate periodic solution is stable.

(ii) If at least the magnitude of one eigenvalue of $D P$ is greater than one (i.e., $\left|\lambda_{i}\right|>1, i \in 1,2, \ldots, 2(s+1)$ ), the approximate periodic solution is unstable.

(iii) The boundaries between stable and unstable periodic flows with higher order singularity give bifurcation and stability conditions with higher order singularity.

The bifurcation conditions are given as follows.

(iv) If $\lambda_{i}=1$ with $\left|\lambda_{j}\right|<1(i, j \in 1,2, \ldots, 2(s+1)$ and $i \neq j)$, the saddle-node bifurcation (SN) occurs.

(v) If $\lambda_{i}=-1$ with $\left|\lambda_{j}\right|<1(i, j \in 1,2, \ldots, 2(s+1)$ and $i \neq j)$, the period-doubling bifurcation (PD) occurs.

(vi) If $\left|\lambda_{i, j}\right|=1$ with $\left|\lambda_{l}\right|<1\left(i, j, l \in 1,2, \ldots, 2(s+1)\right.$ and $\left.\lambda_{i}=\bar{\lambda}_{j}, l \neq i, j\right)$, Neimark bifurcation (NB) occurs.

\section{Analytical predictions of bifurcation trees}

In this section, the complete bifurcation tree of period-1 motion to chaos for the time-delayed, quadratic nonlinear oscillator will be presented through the analytical predictions of period- 1 to period- 4 motions. The corresponding eigenvalue analysis will also be computed for the stability and bifurcation of periodic motions in the time-delay nonlinear systems. Numerical results of periodic motions for the time-delayed system will be illustrated. Consider a set of system parameters as

$$
\alpha_{1}=15.0, \alpha_{2}=5.0, \beta=5.0, \delta=0.05, Q_{0}=4.5
$$

and the time-delay is $\tau=T / 4$ where $T=2 \pi / \Omega$.

The analytical bifurcation trees of period- 1 to period- 4 motions in the time-delayed quadratic oscillator are illustrated in Fig.3 for $\Omega \in(1.885,2.1)$. For $\Omega>2.1$ a simple period-1 motion exists, which will not be presented herein. For $\Omega<1.885$ the complicated unstable periodic motions exist and other stable periodic motion with a short frequency range will not be presented. The prediction of complete bifurcation trees of the period- 1 motion to chaos is presented through the period- 1 to period- 4 motions in the prescribed range of excitation frequency. The solid and dashed curves depict the stable and unstable motions, respectively. The acronyms 'SN', 'PD' and 'NB' represent the saddle node, period doubling, and Neimark bifurcations, respectively. The prediction of displacement and velocity of the periodic nodes $x_{\bmod (k, N)}$ and $y_{\bmod (k, N)}$ for $\bmod (k, N)=0$ varying with excitation frequency are presented in Fig.3(a) and (b), respectively. The period-1, period-2, and period-4 motions are labeled by P-1, P-2, and P-4, respectively. The period-2 motions appears from the PD bifurcations of the period- 1 motions, and period- 4 motion appears from the PD bifurcation of the period-2 motion. The PD bifurcation points for period-1, period-2, period-4 motions are $\Omega \approx 1.89546,1.89554,1.89545,1.89653$, respectively. In addition, there are two saddle-node bifurcation for period- 2 motions at $\Omega \approx 1.89545,1.89653$. The NB and SN bifurcations of a period- 1 motion are at $\Omega \approx 2.0299$ and 2.1079 , respectively.

\section{Numerical Simulations}

Consider the node points of period-m motions as $\mathbf{x}_{k}^{(m)}$ in the quadratic nonlinear oscillator. The approximate expression for period-m motion is determined by the finite Fourier series as

$$
\mathbf{x}^{(m)}(t) \approx \mathbf{a}_{0}^{(m)}+\sum_{j=1}^{M} \mathbf{b}_{j / m} \cos \left(\frac{j}{m} \Omega t\right)+\mathbf{c}_{j / m} \sin \left(\frac{j}{m} \Omega t\right) .
$$



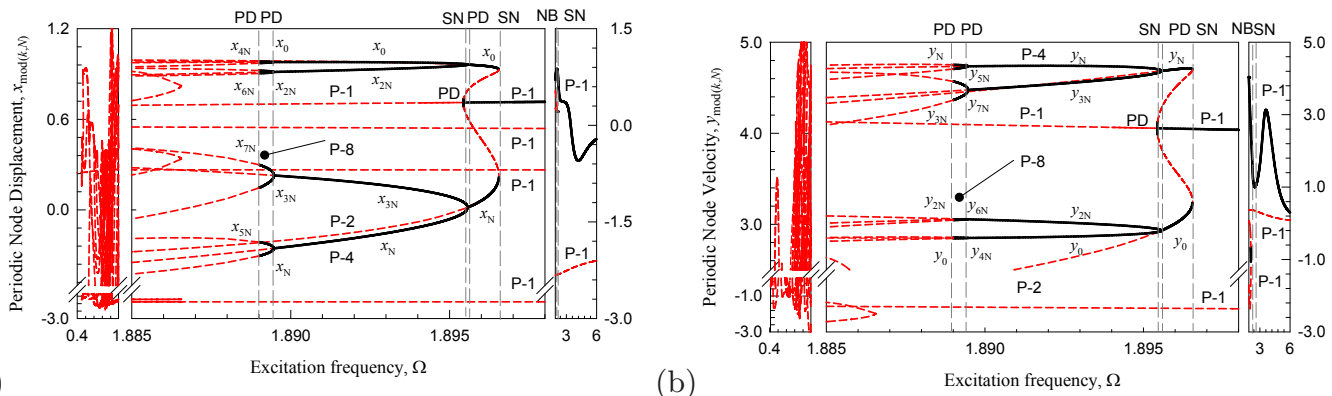

(a)

FiguRE 3. The bifurcation tree of period-1 to period-4 motions varying with excitation frequency. (a) node displacement $x \bmod (k, N)$, (b) node velocity $y \bmod (k, N) \cdot \quad\left(\alpha_{1}=\right.$ 15.0, $\left.\alpha_{2}=5.0, \beta=5.0, \delta=0.05, Q_{0}=4.5, \tau=T / 4\right) . \bmod (k, N)=0$.

There are $(2 M+1)$ unknown vector coefficients of $\mathbf{a}_{0}^{(m)}, \mathbf{b}_{j / m}, \mathbf{c}_{j / m}$. To determine such unknowns, at least we have the given nodes $\mathbf{x}_{k}^{(m)}(k=0,1,2, \ldots, m N)$ with $m N+1 \geq 2 M+1$. In other words, we have $M \leq m N / 2$. The node points $\mathbf{x}_{\mathbf{k}}^{(m)}$ on the period-m motion can be expressed by the finite Fourier series as for $t_{k} \in[0, m T]$,

$$
\begin{aligned}
& \mathbf{x}^{(m)}\left(t_{k}\right) \equiv \mathbf{x}_{k}^{(m)}=\mathbf{a}_{0}^{(m)}+\sum_{j=1}^{m N / 2} \mathbf{b}_{j / m} \cos \left(\frac{j}{m} \Omega t_{k}\right)+\mathbf{c}_{j / m} \sin \left(\frac{j}{m} \Omega t_{k}\right) \\
&=\mathbf{a}_{0}^{(m)}+\sum_{j=1}^{m N / 2} \mathbf{b}_{j / m} \cos \left(\frac{j}{m} \frac{2 k \pi}{N}\right)+\mathbf{c}_{j / m} \sin \left(\frac{j}{m} \frac{2 k \pi}{N}\right) \\
&(k=0,1, \cdots, m N-1)
\end{aligned}
$$

where

$$
\begin{aligned}
& \left.\begin{array}{l}
T=\frac{2 \pi}{\Omega}=N \Delta t ; \Omega t_{k}=\Omega k \Delta t=\frac{2 k \pi}{N} \\
\mathbf{a}_{0}^{(m)}=\frac{1}{m N} \sum_{k=0}^{m-1} \mathbf{x}_{k}^{(m)}, \\
\mathbf{b}_{j / m}=\frac{2}{m N} \sum_{k=1}^{m N-1} \mathbf{x}_{k}^{(m)} \cos \left(k \frac{2 j \pi}{m N}\right), \\
\mathbf{c}_{j / m}=\frac{2}{m N} \sum_{k=1}^{m-1} \mathbf{x}_{k}^{(m)} \sin \left(k \frac{2 j \pi}{m N}\right)
\end{array}\right\}(j=1,2, \cdots, m N / 2)
\end{aligned}
$$

and

$$
\mathbf{a}_{0}^{(m)}=\left(a_{01}^{(m)}, a_{02}^{(m)}\right)^{\mathrm{T}}, \mathbf{b}_{j / m}=\left(b_{j / m 1}, b_{j / m 2}\right)^{\mathrm{T}}, \mathbf{c}_{j / m}=\left(c_{j / m 1}, c_{j / m 2}\right)^{\mathrm{T}}
$$

The harmonic amplitudes and harmonic phases for period-m motion are

$$
\begin{aligned}
& A_{j / m 1}=\sqrt{b_{j / m 1}^{2}+c_{j / m 1}^{2}}, \varphi_{j / m 1}=\arctan \frac{c_{j / m 1}}{b_{j / m 1}}, \\
& A_{j / m 2}=\sqrt{b_{j / m 2}^{2}+c_{j / m 2}^{2}}, \varphi_{j / m 2}=\arctan \frac{c_{j / m 2}}{b_{j / m 2}} .
\end{aligned}
$$

Thus, the approximate expression for period-m motion in Eq.(5.1) is determined by

$$
\mathbf{x}^{(m)}(t) \approx \mathbf{a}_{0}^{(m)}+\sum_{j=1}^{m N / 2} \mathbf{b}_{j / m} \cos \left(\frac{j}{m} \Omega t\right)+\mathbf{c}_{j / m} \sin \left(\frac{j}{m} \Omega t\right) .
$$


The foregoing equation can be expressed as

$$
\left\{\begin{array}{l}
x^{(m)}(t) \\
y^{(m)}(t)
\end{array}\right\} \equiv\left\{\begin{array}{l}
x_{1}^{(m)}(t) \\
x_{2}^{(m)}(t)
\end{array}\right\} \approx\left\{\begin{array}{l}
a_{01}^{(m)} \\
a_{02}^{(m)}
\end{array}\right\}+\sum_{j=1}^{m N / 2}\left\{\begin{array}{c}
A_{j / m 1} \cos \left(\frac{j}{m} \Omega t-\varphi_{j / m 1}\right) \\
A_{j / m 2} \cos \left(\frac{j}{m} \Omega t-\varphi_{j / m 2}\right)
\end{array}\right\} .
$$

For simplicity, harmonic amplitudes of displacement $x^{(m)}(t)$ for period-m motions will be presented. Similarly, the harmonic amplitudes of velocity $y^{(m)}(t)$ for periodic-m motions can also be determined. Thus the displacement can be approximately expressed as

$$
x^{(m)}(t) \approx a_{0}^{(m)}+\sum_{j=1}^{m N / 2} b_{j / m} \cos \left(\frac{j}{m} \Omega t\right)+c_{j / m} \sin \left(\frac{j}{m} \Omega t\right)
$$

and

$$
x^{(m)}(t) \approx a_{0}^{(m)}+\sum_{j=1}^{m N / 2} A_{j / m} \cos \left(\frac{j}{m} \Omega t-\varphi_{j / m}\right)
$$

where

$$
A_{j / m}=\sqrt{b_{j / m}^{2}+c_{j / m}^{2}}, \varphi_{j / m}=\arctan \frac{c_{j / m}}{b_{j / m}} .
$$

From the analytical prediction, the initial condition will be obtained for numerical computation of periodic motion, and the corresponding harmonic amplitudes distributions(or spectrums) will be presented to show harmonic effects on periodic motions. The system parameters are used in Eq.(4.1). Numerical and analytical results are presented by solid curves and symbols, respectively. The initial time-delay are presented through green circular symbols. The delay-initial-starting and delay-initial-finishing points are 'D.I.S.' and 'D.I.F.', respectively.

From the analytical prediction, the displacement, velocity, trajectory and harmonic amplitude for the stable period- 1 motion are presented at $\Omega=5.36$. The initial condition is $x_{0} \approx-0.302967$ and $\dot{x}_{0} \approx$ 0.563768. The time-histories of displacement and velocity are presented in Fig.4(a) and (b), respectively. One period in the corresponding time histories is labeled. The initial time-delay point is depicted by the green symbols. The numerical solution of the stable period-1 motion is presented by the solid curve, while the analytical prediction is presented by red symbols. The trajectory of the simple periodic motion is given in Fig.4(c), and the corresponding harmonic amplitude is placed in Fig.4(d). The harmonic amplitudes are: $a_{0} \approx-0.230, A_{1} \approx 0.3018, A_{2} \approx 2.3936 e-3, A_{3} \approx 1.4813 e-5$ and $A_{4} \approx 8.1513 e-8$. With harmonic order increase, the harmonic amplitude decreases dramatically. For instance, $A_{7} \approx 1.0311 e-14$. For this periodic motion, one can use two harmonic terms to approximate such a simple periodic motion.

The trajectory and amplitude spectrum of two stable period-1 motions for the timed-delayed, quadratic nonlinear oscillator are presented in Fig.5 for $\Omega=2$ and 2.1078 on the two different branches. The initial conditions are $\left(x_{0}, \dot{x}_{0}\right) \approx(0.859503,3.203165)$ and $\left(x_{0}, \dot{x}_{0}\right) \approx(0.206385,-1.039637)$ for $\Omega=2$ and 2.1078, respectively. In Fig.5(a), analytical and numerical trajectories are presented for $\Omega=2$, and the initial time-delayed responses in phase plane is illustrated. The period-1 motion has two cycles. The green points give the initial time-delay range in phase plane. To know the harmonic effect on periodic motion, the analytical harmonic amplitude distribution is presented in Fig.5(b). The main harmonic amplitudes are $a_{0} \approx-0.4486, A_{1} \approx 0.3087, A_{2} \approx 1.1352, A_{3} \approx 0.0707, A_{4} \approx 0.0562, A_{5} \approx$ $5.4754 \mathrm{e}-3, A_{6} \approx 2.5547 \mathrm{e}-3, A_{7} \approx 2.9619 \mathrm{e}-4$. The other harmonic amplitudes are $A_{8} \approx 9.3943 \mathrm{e}-5, A_{9} \approx$ $1.3609 \mathrm{e}-5, A_{10} \approx 3.5276 \mathrm{e}-6, A_{\mathrm{k}} \in\left(10^{-14}, 10^{-7}\right)$ for $k=11,12, \ldots, 20$. In addition, period- 1 motion exists on another solution branch. The analytical and numerical trajectories are presented in Fig.5(c) for $\Omega=2.1078$. The period- 1 motion is very small compared to the period- 1 motion at $\Omega=2$ on the another branch. The quantity level of the harmonic amplitudes is smaller than the aforementioned period-1 motion, i.e., $a_{0} \approx-0.1810, A_{1} \approx 0.4689, A_{2} \approx 0.3532, A_{3} \approx 0.0312, A_{4} \approx 3.8805 \mathrm{e}-3, A_{5} \approx$ 
$6.5834 \mathrm{e}-4, A_{6} \approx 2.1718 \mathrm{e}-5, A_{7} \approx 8.9109 \mathrm{e}-6$. The other harmonic amplitudes are $A_{8} \approx 1.9892 \mathrm{e}-7, A_{9} \approx$ $9.0871 \mathrm{e}-8, A_{10} \approx 6.1769 \mathrm{e}-9, A_{k} \in\left(10^{-14}, 10^{-9}\right)$ for $k=11,12, \ldots, 20$. For this period-1 motion, one should use at least 7 harmonic terms to approximately discribe the periodic motion. For more accurate results, 20 harmonic terms should be used in the approximate anayltical solution.

(a)
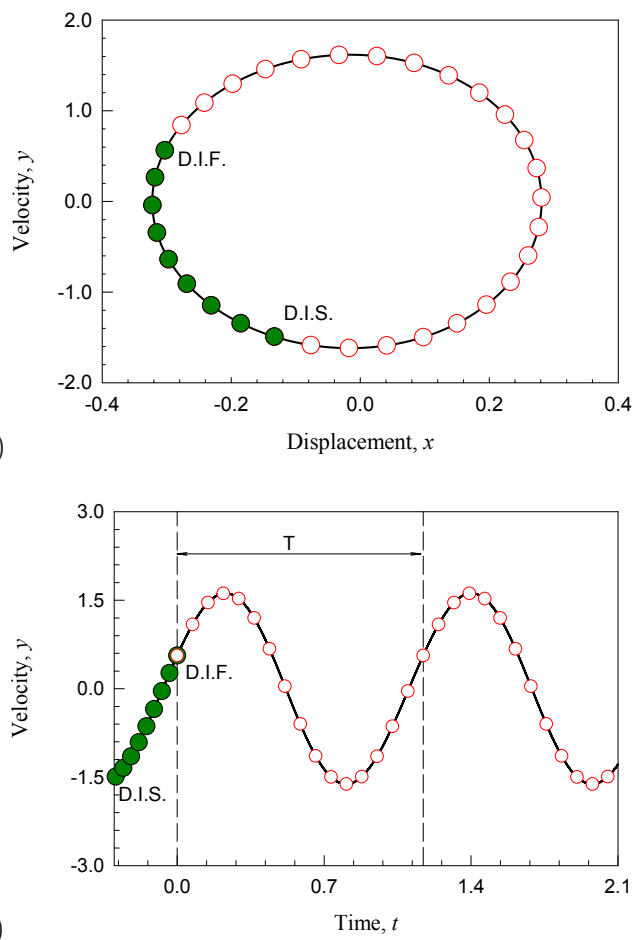

(b)
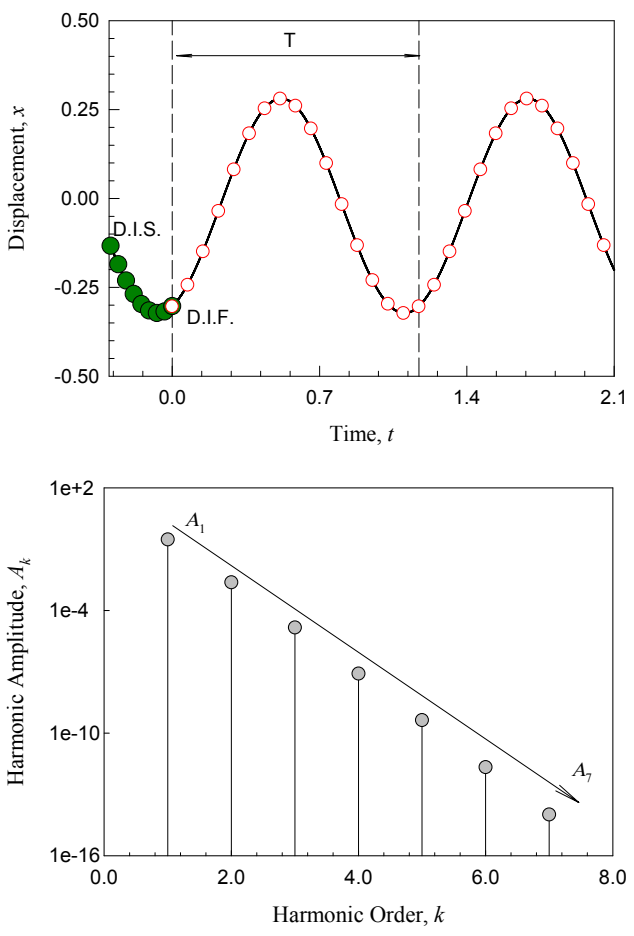

Figure 4. Stable simple period-1 motion $(\Omega=5.36)$. (a) trajectory, (b) displacement, (c) velocity, (d) harmonic amplitude. $x_{0} \approx-0.302967, \dot{x}_{0} \approx 0.563768\left(\alpha_{1}=15.0, \alpha_{2}=\right.$ $\left.5.0, \beta=5.0, \delta=0.05, Q_{0}=4.5, \tau=T / 4\right)$.

The trajectories and amplitudes for stable period-2 motions for the timed-delayed, quadratic nonlinear oscillator are presented in Fig.6 for $\Omega=1.89544$. The harmonic phase of the stable period-2 motions is $\varphi_{k / 2}^{(1)}$, which will not be presented herein for simplicity. In Fig.6(a), analytical and numerical trajectories of period- 2 motion are presented for the initial condition $\left(x_{0}, \dot{x}_{0}\right) \approx(0.740611,3.955127)$ through the solid curve (numerical) and circular symbols (analytical), and the initial time-delayed responses in phase plane are illustrated through the blue circular symbols. The trajectory of period-2 motion is the doubling period-1 motion, and the two curves are very close. In Fig.6(b), the analytical harmonic amplitude distribution is presented from the analytical prediction of implicit mapping structure. The harmonic amplitudes are $a_{0}^{(2)} \approx-0.621167, A_{1 / 2} \approx 0.011365, A_{1} \approx 0.309662, A_{3 / 2} \approx 0.014120, A_{2} \approx$ $1.266238, A_{5 / 2} \approx 7.723310 \mathrm{e}-3, A_{3} \approx 0.086476, A_{7 / 2} \approx 2.004510 \mathrm{e}-3, A_{4} \approx 0.076306, A_{9 / 2} \approx$ 8.701976e- $4, A_{5} \approx 8.266150 \mathrm{e}-3, A_{11 / 2} \approx 1.666896 \mathrm{e}-4$ and $A_{6} \approx 4.294737 \mathrm{e}-3$. The other harmonic amplitudes are $A_{k / 2} \in\left(10^{-13}, 10^{-4}\right)$ for $k=13,14, \ldots, 40$. The harmonic phase angles $\varphi_{k / 2}^{(1)}$ with different initial conditions can be obtained by $\varphi_{k / 2}^{(2)}=\bmod \left(\varphi_{k / 2}^{(1)}+k \pi, 2 \pi\right)(k=1,2, \ldots, 40)$. Such a periodic motion can be approximated by 40 harmonic terms with the accuracy of $10^{-13}$. 

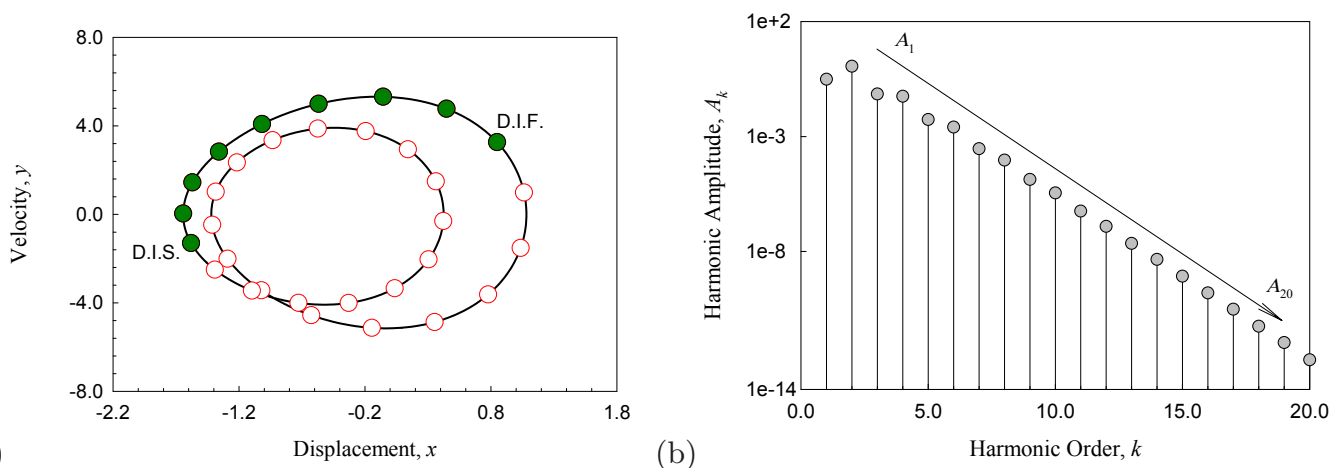

(a)

(b)
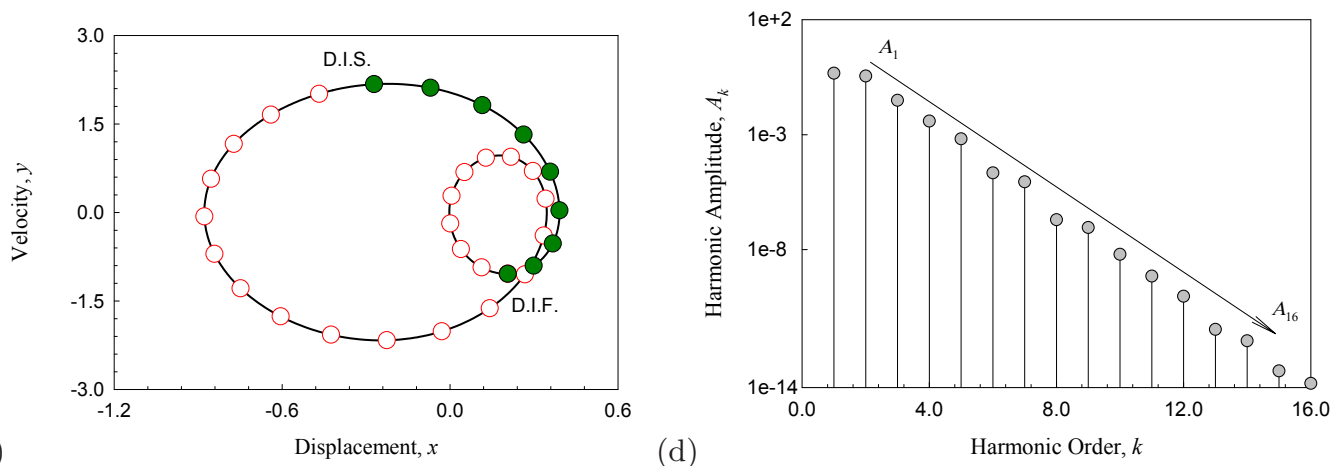

Figure 5. Two stable period-1 motions: (a) Trajectory, (b) harmonic amplitude for $(\Omega=2.0)$ with initial condition $x_{0} \approx 0.859503, \dot{x}_{0} \approx 3.203165 ;$ (c) Trajectory, $(\mathrm{d})$ harmonic amplitude for $(\Omega=2.1078)$ with initial condition $x_{0} \approx 0.206385, \dot{x}_{0} \approx$ -1.039637. $\left(\alpha_{1}=15.0, \alpha_{2}=5.0, \beta=5.0, \delta=0.05, Q_{0}=4.5, \tau=T / 4\right)$.

The trajectory and amplitude spectrum of an unstable period-2 motion in the timed-delayed oscillator with the initial condition is $\left(x_{0}, \dot{x}_{0}\right) \approx(0.869038,3.564403)$ are in Fig.7 for $\Omega=1.89606$. The unstable period-2 motion has harmonic phase $\varphi_{k / 2}^{(1)}$. To reduce abundant plots, the harmonic phase angles will not be presented herein. As before in Fig.7(a), analytical and numerical trajectories of unstable period- 2 motion are presented through the solid curve (numerical) and circular symbols (analytical), and the initial time-delayed responses in phase plane is illustrated through the green circular symbols. Once the computational time becomes longer, the unstable period- 2 motion goes to the stable period-1 motion which is near such an unstable period-2 motion. The stable period-1 motion is presented by the thin dashed curve and the corresponding analytical prediction is presented by symbols "x". The analytical harmonic amplitude distribution is presented in Fig.7(b). The harmonic amplitudes are $a_{0}^{(2)} \approx-0.6141, A_{1 / 2} \approx 0.1387, A_{1} \approx 0.3177, A_{3 / 2} \approx 0.1682, A_{2} \approx 1.2376, A_{5 / 2} \approx 0.0942, A_{3} \approx$ $0.0864, A_{7 / 2} \approx 0.0229, A_{4} \approx 0.0712, A_{9 / 2} \approx 0.0104, A_{5} \approx 8.0013 \mathrm{e}-3, A_{11 / 2} \approx 1.7899 \mathrm{e}-3, A_{6} \approx$ $3.7385 \mathrm{e}-3, A_{13 / 2} \approx 8.2743 \mathrm{e}-4, A_{7} \approx 5.0570 \mathrm{e}-4$. The other harmonic amplitudes are $A_{k / 2} \in\left(10^{-13}, 10^{-4}\right)$ for $k=15,16, \ldots, 40$. With different initial conditions, the harmonic phase angles of another periodic-2 motion are $\varphi_{k / 2}^{(2)}=\bmod \left(\varphi_{k / 2}^{(1)}+k \pi, 2 \pi\right)$. The unstable period-2 motion can be approximated by 40 harmonic terms in the analytical solutions as in Luo and Jin[12]. For the period-2 motions, the harmonic distributions in Fig.7 are different from those in Fig.6. The harmonic amplitudes for period-2 motions only play significant contributions in the period-2 motions. Thus, the curves in the trajectory in phase plane will be separated, which are different from the period-2 motion in Fig.6. 
The trajectories and amplitudes for stable period-2 motions for the timed-delayed, quadratic nonlinear oscillator are presented in Fig. 8 for $\Omega=1.89592$. In Fig.8(a), analytical and numerical trajectories of period-2 motion are presented for the initial condition $\left(x_{0}, \dot{x}_{0}\right) \approx(0.96692,2.923095)$. In Fig.8(b), the analytical harmonic amplitude distribution is presented from the analytical prediction of implicit mapping. The harmonic amplitudes are $a_{0}^{(2)} \approx-0.590233, A_{1 / 2} \approx 0.305991, A_{1} \approx 0.363191, A_{3 / 2} \approx$ $0.339724, A_{2} \approx 1.126255, A_{5 / 2} \approx 0.205169, A_{3} \approx 0.089182, A_{7 / 2} \approx 0.038089, A_{4} \approx 0.053258, A_{9 / 2} \approx$ $0.020931, A_{5} \approx 7.229025 e-3, A_{11 / 2} \approx 2.234087 e-3$ and $A_{6} \approx 2.035762 e-3$. The other harmonic amplitudes are $A_{k / 2} \in\left(10^{-13}, 10^{-3}\right)$ for $k=13,14, \ldots, 40$. The harmonic phase is $\varphi_{k / 2}^{(1)}$. The harmonic phase angle for another period-2 motions at the same excitation frequency is $\varphi_{k / 2}^{(2)}=\bmod \left(\varphi_{k / 2}^{(1)}+k \pi, 2 \pi\right)$. The period-2 motion can be also approximated by the 40 harmonic terms, and the harmonic amplitude distributions are similar for the period-2 motions in Fig. 6 and 7. However, the four cycles of the period-2 motion trajectory in phase plane are different from these period-2 motions in Fig.6 and 7.
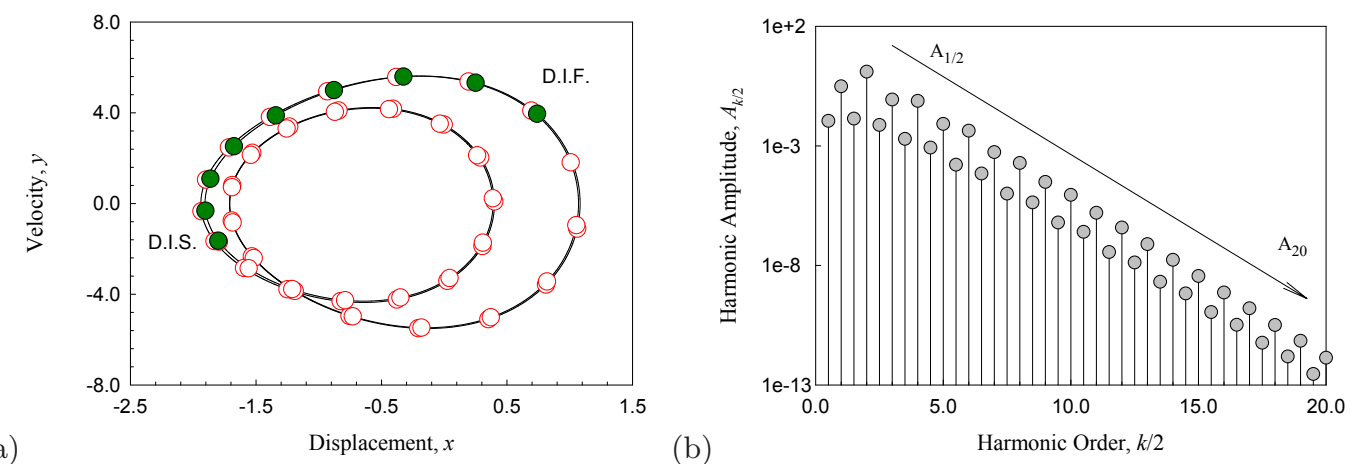

Figure 6 . A pair of stable period-2 motions $(\Omega=1.89544)$ : branch-1: (a) trajectory and $(\mathrm{b})$ harmonic amplitude $\left(x_{0} \approx 0.740611\right.$, and $\left.\dot{x}_{0} \approx 3.955127\right) . \quad\left(\alpha_{1}=15.0, \alpha_{2}=\right.$ $\left.5.0, \beta=5.0, \delta=0.05, Q_{0}=4.5, \tau=T / 4\right)$.
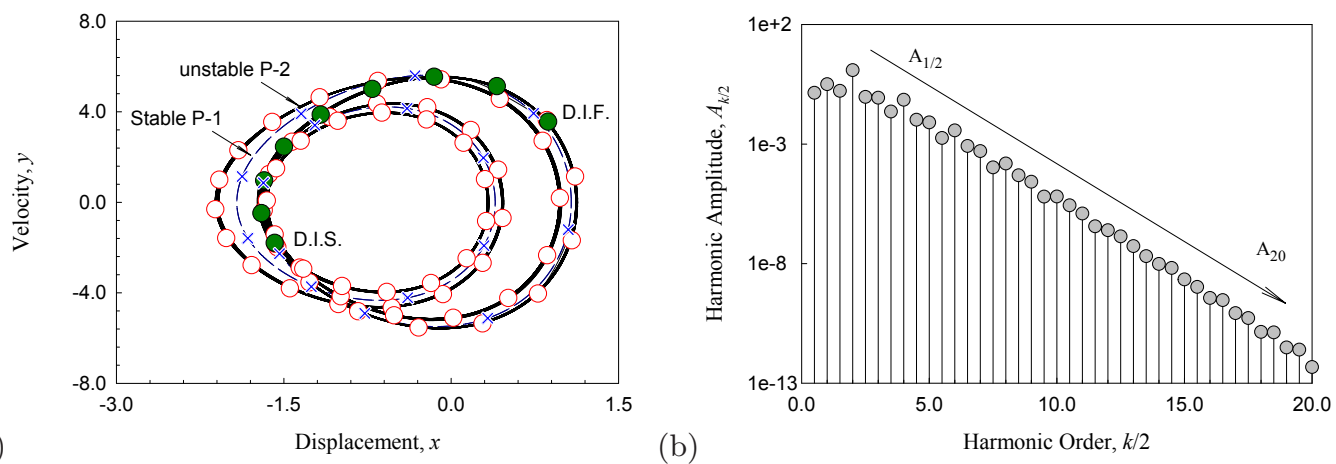

Figure 7. Unstable period-2 motion to stable period-1 motion $(\Omega=1.89606)$, (a) trajectory and $(\mathrm{b})$ harmonic amplitude $\left(x_{0} \approx 0.869038, \dot{x}_{0} \approx 3.564403\right) .\left(\alpha_{1}=15.0, \alpha_{2}=\right.$ $\left.5.0, \beta=5.0, \delta=0.05, Q_{0}=4.5, \tau=T / 4\right)$. 

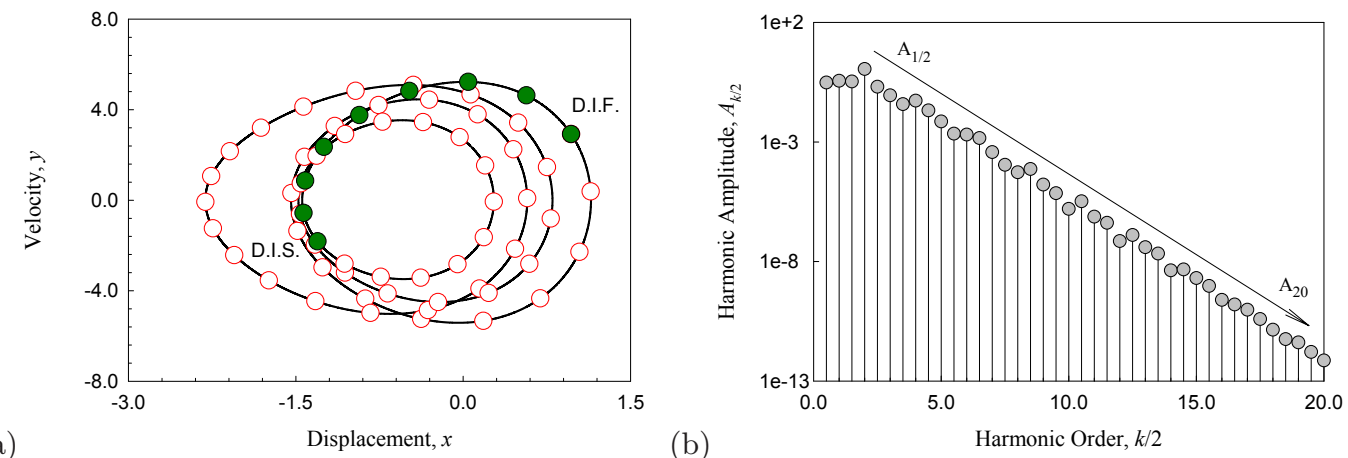

FiguRE 8. Stable period-2 motions $(\Omega=1.89592)$ : (a) trajectory and (b) harmonic amplitude $\left(x_{0} \approx 0.966920\right.$, and $\left.\dot{x}_{0} \approx 2.923095\right) .\left(\alpha_{1}=15.0, \alpha_{2}=5.0, \beta=5.0, \delta=\right.$ $\left.0.05, Q_{0}=4.5, \tau=T / 4\right)$.

The trajectories and amplitude spectrum for stable period-4 motions for the time-delayed, quadratic nonlinear oscillator are presented in Fig.9 for $\Omega=1.89545$. The initial condition $\left(x_{0}, \dot{x}_{0}\right) \approx$ $(0.976007,2.845607)$ is used first, and analytical and numerical trajectories are also presented in Fig.9(a). The trajectory of period- 4 motion is close to the period- 2 motion in Fig. 8 because the period- 4 motion is obtained by the period-doubling of period-2 motion. To know harmonic effects on the complexity of stable period-4 motion, the harmonic amplitudes should be discussed. Thus, in Fig.9(b), the analytical harmonic amplitude distribution is presented. For instance, $a_{0}^{(4)} \approx-0.5878, A_{1 / 4} \approx 0.0107, A_{1 / 2} \approx 0.3210, A_{3 / 4} \approx$ $4.5519 \mathrm{e}-3, A_{1} \approx 0.3701, A_{5 / 4} \approx 3.9566 \mathrm{e}-3, A_{3 / 2} \approx 0.3524, A_{7 / 4} \approx 0.0173, A_{2} \approx 1.1120, A_{9 / 4} \approx$ $0.0125, A_{5 / 2} \approx 0.2147, A_{11 / 4} \approx 2.1774 \mathrm{e}-3, A_{3} \approx 0.0897, A_{13 / 4} \approx 1.3076 \mathrm{e}-3, A_{7 / 2} \approx 0.0384, A_{15 / 4} \approx$ $1.6172 \mathrm{e}-3, A_{4} \approx 0.0512, A_{17 / 4} \approx 1.3049 \mathrm{e}-3, A_{9 / 2} \approx 0.0217, A_{19 / 4} \approx 2.2776 \mathrm{e}-4, A_{5} \approx 7.1471 \mathrm{e}-3, A_{21 / 4} \approx$ $1.7302 \mathrm{e}-4, A_{11 / 2} \approx 2.1527 \mathrm{e}-3, A_{23 / 4} \approx 7.6535 \mathrm{e}-5, A_{6} \approx 1.8696 \mathrm{e}-3, A_{25 / 4} \approx 9.8349 \mathrm{e}-5, A_{13 / 2} \approx$ $1.4883 \mathrm{e}-3, A_{27 / 4} \approx 1.8010 \mathrm{e}-5, A_{7} \approx 3.6609 \mathrm{e}-4$. The other harmonic amplitudes are $A_{k / 4} \in\left(10^{-13}, 10^{-4}\right)$ for $k=29,30, \cdots, 80$. For other initial conditions, $\varphi_{k / 4}^{(j)}=\bmod \left(\varphi_{k / 4}^{(1)}+(j-1) k \pi / 2,2 \pi\right),(j=2,3,4,5)$. Such period- 4 motion can be approximated by 80 harmonic terms in the analytic solution in Luo and Jin[12]. The harmonic amplitudes related to the period-4 motions only are very small, which causes the period-4 motion close to period-2 motion in Fig.8.

To show the complexity of period- 8 motion, the trajectories and amplitude spectrums for stable period8 motions for the timed-delayed, quadratic nonlinear oscillator are presented in Fig.10 for $\Omega=1.8893$. The initial condition is $\left(x_{0}, \dot{x}_{0}\right) \approx(0.991002,2.773226)$ for the first branch of periodi- 8 motion, and the corresponding analytical and numerical trajectories of such a period-8 motion are presented in Fig.10(a). The trajectory of the period- 8 motion becomes more complicated than period-1, period-2, and period- 4 motions. For this motion complexity, the harmonic amplitudes should be discussed. Thus, in Fig.10(b), the corresponding analytical harmonic amplitude distribution is presented by implicit mapping. $a_{0}^{(4)} \approx$ $-0.5948, A_{1 / 8} \approx 5.4408 \mathrm{e}-3, A_{1 / 4} \approx 0.0755, A_{5 / 8} \approx 0.0109, A_{1 / 2} \approx 0.3234, A_{5 / 8} \approx 7.9519 \mathrm{e}-3, A_{3 / 4} \approx$ $0.0333, A_{7 / 8} \approx 4.2828 \mathrm{e}-4, A_{1} \approx 0.3760, A_{9 / 8} \approx 2.0685 \mathrm{e}-3, A_{5 / 4} \approx 0.0272, A_{11 / 8} \approx 7.6828 \mathrm{e}-3, A_{3 / 2} \approx$ $0.3506, A_{13 / 8} \approx 0.0125, A_{7 / 4} \approx 0.1222, A_{15 / 8} \approx 0.0161, A_{2} \approx 1.1012, A_{17 / 8} \approx 6.4813 \mathrm{e}-3, A_{9 / 4} \approx$ $0.0873, A_{19 / 8} \approx 9.9291 \mathrm{e}-3, A_{5 / 2} \approx 0.2144, A_{21 / 8} \approx 3.7565 \mathrm{e}-3, A_{11 / 4} \approx 0.0161, A_{23 / 8} \approx 1.1102 \mathrm{e}-3, A_{3} \approx$ $0.0905, A_{25 / 8} \approx 9.3439 \mathrm{e}-4, A_{13 / 4} \approx 8.9772 \mathrm{e}-3, \quad A_{27 / 8} \approx 1.0048 \mathrm{e}-3, A_{7 / 2} \approx 0.0369, A_{29 / 8} \approx$ $1.1487 \mathrm{e}-3, \quad A_{15 / 4} \approx 0.0113, A_{31 / 8} \approx 1.8189 \mathrm{e}-3, \quad A_{4} \approx 0.0502, \quad A_{33 / 8} \approx 5.6598 \mathrm{e}-4, A_{17 / 4} \approx$ $8.9936 \mathrm{e}-3, \quad A_{35 / 8} \approx 1.0327 \mathrm{e}-3, A_{9 / 2} \approx 0.0212, A_{37 / 8} \approx 3.3757 \mathrm{e}-4, A_{19 / 4} \approx 1.6557 \mathrm{e}-3, A_{39 / 8} \approx$ $1.7837 \mathrm{e}-4, A_{5} \approx 7.2107 \mathrm{e}-3, A_{41 / 8} \approx 8.4335 \mathrm{e}-5, \quad A_{21 / 4} \approx 1.1870 \mathrm{e}-3, A_{43 / 8} \approx 1.2386 \mathrm{e}-4, A_{11 / 2} \approx$ $1.9499 \mathrm{e}-3, A_{45 / 8} \approx 6.3985 \mathrm{e}-5, A_{23 / 4} \approx 5.1395 \mathrm{e}-4, A_{47 / 8} \approx 1.2247 \mathrm{e}-4, A_{6} \approx 1.8854 \mathrm{e}-3, A_{49 / 8} \approx$ $4.3099 \mathrm{e}-5, A_{25 / 4} \approx 6.6595 \mathrm{e}-4, A_{51 / 8} \approx 7.6026 \mathrm{e}-5, A_{13 / 2} \approx 1.4134 \mathrm{e}-3, A_{53 / 8} \approx 2.2289 \mathrm{e}-5, A_{27 / 4} \approx$ 

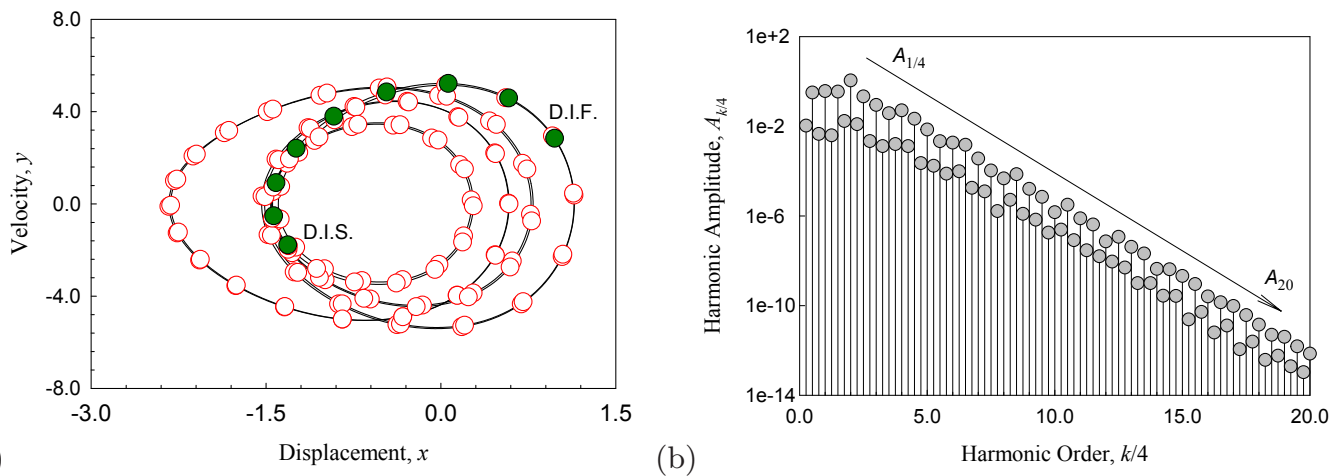

FiguRE 9. Stable period-4 motions $(\Omega=1.89545)$ : (a) trajectory and (b) harmonic amplitude $\left(x_{0} \approx 0.976007, \dot{x}_{0} \approx 2.845607\right) ;\left(\alpha_{1}=15.0, \alpha_{2}=5.0, \beta=5.0, \delta=0.05, Q_{0}=\right.$ $4.5, \tau=T / 4)$.

$1.2372 \mathrm{e}-4, A_{55 / 8} \approx 1.6518 \mathrm{e}-5, A_{7} \approx 3.8095 \mathrm{e}-4$. The other harmonic amplitudes are $A_{k / 8} \in\left(10^{-13}, 10^{-4}\right)$ for $k=29,30, \cdots, 160$. For other initial conditions, $\varphi_{k / 8}^{(j)}=\bmod \left(\varphi_{k / 8}^{(1)}+(j-1) k \pi / 4,2 \pi\right)(j=2,3, \ldots, 8,9)$. The corresponding harmonic amplitudes are same. Such a period-8 motion can be approximated by 160 harmonic terms to achieve.
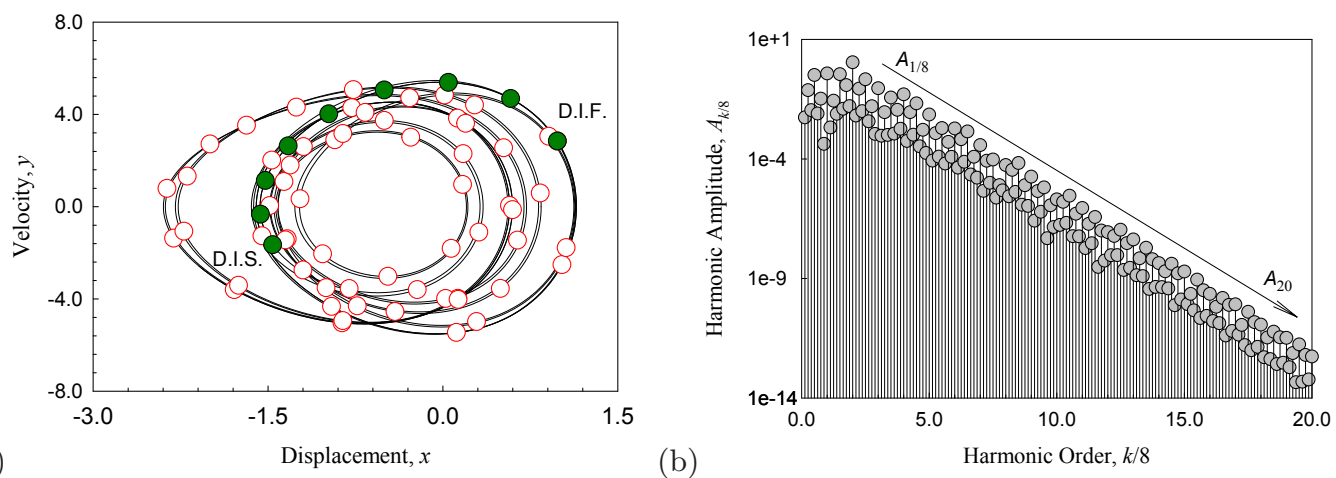

Figure 10. Stable period-8 motions $(\Omega=1.8893):(a)$ trajectory and (b) harmonic amplitude $\left(x_{0} \approx 0.991002, \dot{x}_{0} \approx 2.773226\right) . \quad\left(\alpha_{1}=15.0, \alpha_{2}=5.0, \beta=5.0, \delta=\right.$ $\left.0.05, Q_{0}=4.5, \tau=T / 4\right)$.

\section{Conclusions}

In this paper, analytical prediction of periodic motions in a periodically forced, damped, quadratic nonlinear oscillator with time-delayed displacement were computed through implicit discrete mappings. The time-delay nodes of periodic motions were interpolated by the non-time-delayed nodes to form the implicit mappings. From mapping structures of periodic motion, bifurcation trees of periodic motions were obtained analytically, and the corresponding stability and bifurcation were completed through eigenvalue analysis. From the analytical prediction, numerical results of periodic motions were illustrated to verify 
such an analytical prediction. From the semi-analytical method, the complexity of periodic motions in the time-delayed, quadratic nonlinear systems was investigated, which matches very well with the analytical solutions in Luo and Jin [12]. The method presented in this paper can be applied to other time-delayed, nonlinear dynamical systems.

\section{References}

[1] J. L. Lagrange. Mecanique Analytique. vol. 2, 1788.

[2] H. Poincare. Methodes Nouvelles de la Mecanique Celeste. Vol. 3, Gauthier-Villars: Paris, 1899.

[3] B. van der Pol. A theory of the amplitude of free and forced triode vibrations. Radio Review 1(1920), 701-710, 754-762.

[4] P. Fatou. Sur le mouvement d'un systeme soumis 'a des forces a courte periode. Bulletin de la Société Mathématique de France. 56(1928), 98-139.

[5] N.M. Krylov, N.N. Bogolyubov. Methodes approchees de la mecanique nonlineaire dans leurs application a l'Aeetude de la perturbation des mouvements periodiques de divers phenomenes de resonance s'y rapportant. Academie des Sciences d'U-kraine, Kiev(French), 1935.

[6] A.C.J. Luo. Continuous Dynamical Systems. HEP/LH Scientific: Beijing/Glen Carbon, 2012.

[7] A.C.J. Luo, J.Z. Huang. Approximate solutions of periodic motions in nonlinear systems via a generalized harmonic balance. Journal of Vibration and Control 18(2012), No. 11, 1661-1871.

[8] A.C.J. Luo, J.Z. Huang. Analytical dynamics of period-m flows and chaos in nonlinear systems. International Journal of Bifurcation and Chaos, 22(2012), No. 4, Article No. 1250093 (29 pages).

[9] A.C.J. Luo, J.Z. Huang. Analytical routines of period-1 motions to chaos in a periodically forced Duffing oscillator with twin-well potential. Journal of Applied Nonlinear Dynamics, 1(2012), No. 1, 73-108.

[10] A.C.J. Luo, J.Z. Huang. Unstable and stable period-m motions in a twin-well potential Duffing oscillator. Discontinuity, Nonlinearity and Complexity 1(2012), No. 3, 113-145.

[11] A.C.J. Luo. Analytical solutions of periodic motions in dynamical systems with/without time-delay. International Journal of Dynamics and Control 1(2013), No. 4, 330-359.

[12] A.C.J. Luo, H.X. Jin. Bifurcation trees of period-m motion to chaos in a Time-delayed, quadratic nonlinear oscillator under a periodic excitation. Discontinuity, Nonlinearity, and Complexity 3(2014), No. 1, 87-107.

[13] A.C.J. Luo, H.X. Jin. Complex period-1 motions of a periodically forced Duffing oscillator with a time-delay feedback. International Journal of Dynamics and Control, 3(2015), No. 4, 325-340.

[14] A.C.J. Luo, A.C.J. Jin. Period-m motions to chaos in a periodically forced Duffing oscillator with a time-delay feedback. International Journal of Bifurcation and Chaos 24(2014), No. 10, Article No.1450126 (20 pages).

[15] A.C.J Luo. The mapping dynamics of periodic motions for a three-piecewise linear system under a periodic excitation. Journal of Sound and Vibration 283(2005), No. 3-5, 723-748.

[16] A.C.J, Luo. Regularity and Complexity in Dynamical Systems. Springer: New York, 2012.

[17] A.C.J. Luo. Periodic flows in nonlinear dynamical systems based on discrete implicit maps. International Journal of Bifurcation and Chaos, 25(2015), No. 3, Artical No. 1550044 (62 pages).

[18] A.C.J Luo, Y. Guo. A semi-analytical prediction of periodic motions in Duffing oscillator through mapping structures. Discontinuity, Nonlinearity and Complexity, 4 (2015), No. 2, 121-150. 